\title{
miRNA expression profile of colon cancer stem cells compared to non-stem cells using the SW1116 cell line
}

\author{
YANTIAN FANG $^{1 *}$, JIANBIN XIANG $^{1 *}$, ZONGYOU CHEN $^{1}$, XIAODONG GU ${ }^{1}$, \\ ZHENGYANG LI $^{1}$, FENG TANG ${ }^{2}$ and ZHONGWEN ZHOU ${ }^{2}$ \\ Departments of ${ }^{1}$ General Surgery and ${ }^{2}$ Pathology, Huashan Hospital Affiliated to Fudan University, \\ Shanghai 200040, P.R. China
}

Received July 2, 2012; Accepted September 4, 2012

DOI: $10.3892 / o r .2012 .2054$

\begin{abstract}
Colorectal cancer (CRC) is one of the major causes of cancer-related mortality worldwide. Recent studies revealed that there is a relationship between CRC occurrence and microRNA (miRNA) function. Stem cells are a type of cells that have the ability to self-renew and to proliferate extensively while maintaining the undifferentiated state. Cancer stem cells (CSCs) are closely linked to tumor recurrence and metastasis. To this end, we evaluated the miRNA expression differences between colon CSCs and non-stem cells using the SW1116 cell line, to determine the relationship between tumor stem cells and tumor biological behavior. We isolated populations of colon CSCs with the $\mathrm{CD} 133^{+} / \mathrm{CD} 44^{+}$and CD133\% CD44- surface phenotype from a human SW1116 colon adenocarcinoma cell line using flow cytometry. The expression of miRNA and mRNA of both sets of cells was examined with miRNA and mRNA arrays. Bioinformatic methods were used to analyze microarray results. We completed gene ontology analysis, pathway analysis, miRNA target gene prediction with databases. We identified a colon stem cell miRNA expression profile comprising 31 upregulated and 31 downregulated miRNAs, such as miR29a, miR29b, miR449b and miR4524. Some of these differentially expressed miRNAs may be involved in the regulation of stem cell differentiation. Gene ontology and pathway analyses showed that the differences are closely related to the function of the cell cycle, cell differentiation, signaling pathway, cytoskeletal proteins and cell-matrix adhesion in colon cancer stem cells. We found that miRNAs play an important role in regulating the expression of colon CSC characteristics. By regulating the expression of CSC signaling pathways, cytoskeleton and membrane proteins,
\end{abstract}

Correspondence to: Professor Zongyou Chen, Department of General Surgery, Huashan Hospital Affiliated to Fudan University, 12 Wulumuqizhong Road, Shanghai 200040, P.R. China

E-mail: zongyouchen@hotmail.com

*Contributed equally

Key words: colon cancer, stem cell, gene ontology, microRNA
miRNAs give tumor stem cells the macrobiological behavior of recurrence and metastasis. This study provides a new perspective on CRC metastasis and recurrence.

\section{Introduction}

Colorectal cancer (CRC) is the third leading cause of cancer-related mortality worldwide (1) and has created new challenges to the methods and tools of treatment. CRC is often the result of a combination of environmental and genetic mutations, accompanied by a variety of gene expression profile changes (2-4). Genetic mutations, including point mutations, chromosomal translocation and gene amplification, result in oncogenes and tumor suppressor gene mutations involved in cancer development (2). Tumor cells are parts of tissues that have lost normal regulation of their growth, resulting in clonal dysplasia and the formation of neoplasm, following the impact of a variety of carcinogenic factors. Inappropriate expression of tumor-suppressor genes or oncogenes is regarded the principal cause of tumorigenesis.

The biological behavior of cancer, including carcinogenesis and functional heterogeneity, can be explained by the cancer stem cell (CSC) hypothesis. According to this model, CSCs, which exhibit stem-like features, are involved in tumor formation, proliferation, differentiation, metastasis and resistance to therapy (5-7). Through their ability of self-renewal and unlimited proliferation, tumor stem cells maintain the vitality of the tumor cell population, and through their increased movement and migration, tumor stem cells make cell metastasis possible. With a variety of drug-resistant molecules, tumor stem cells allow the tumor to become non-sensitive to external physical and chemical factors. At the same time, numerous studies have indicated that some cytokines, protein-coding genes and its products are involved in the maintenance of the biological characteristics of CSCs (8-10). However, the underlying mechanisms remain largely unknown.

microRNAs (miRNAs), approximately 18-24 nt in length, are a class of non-coding single-stranded RNA molecules in eukaryotes. miRNAs regulate their target gene expression through post-transcriptional regulation, which includes mediating degradation of target mRNA, inhibiting target mRNA translation through complementary combining with the target mRNA 3'-untranslated region (UTR) completely/incompletely 
(11-14). Similar to other transcription factors, miRNAs are adjustment factors which can determine cell fate. miRNAs and other post-transcriptional regulatory mechanisms are regarded the mechanisms that control gene expression $(11,15,16)$.

In recent years, the combination of computer science, information technology, mathematical theory and gene chip technology, has given rise to an interdisciplinary science, bioinformatics. Bioinformatics includes biological data handling, processing of the genetic and physical map, nucleotide and amino acid sequence analysis, the discovery of new genes and protein structure prediction. With bioinformatics we can increase our knowledge of genes, and from our previous understanding of a single gene, we can now examine the genes in the whole genome level organizational structure and information structure, and examine the mutual relationships between gene location, structure and function.

However, the reasons underlying the generation, development, recurrence and metastasis of CRC remain unclear and the role of stem cells in the tumor biological processes has yet to be fully elucidated. Using a combination of bio-chip and computer bioinformatics, herein we report a comprehensive analysis of stem cell properties in the CRC cell line.

\section{Materials and methods}

Cell culture and tissues. The SW1116 human CRC cell line was obtained from the Shanghai Chinese Academy of Sciences (CAS). The CRC cells were carefully cultured in RPMI-1640 medium containing $10 \%$ fetal bovine serum, $50 \mathrm{U} / \mathrm{ml}$ penicillin and $50 \mu \mathrm{g} / \mathrm{ml}$ streptomycin. Cells were cultured at $37^{\circ} \mathrm{C}$, in a $5 \% \mathrm{CO}_{2}$ atmosphere with $95 \%$ humidity.

We separated $\mathrm{CD} 133^{+} \mathrm{CD} 44^{+}$-positive and $\mathrm{CD} 133^{-} \mathrm{CD} 44$ -negative cells using flow cytometry. $\mathrm{CD} 133^{+} \mathrm{CD} 44^{+}$-positive cells were cultured in serum-free DMEM/F12 with EGF $(10 \mathrm{ng} / \mathrm{ml})$ and $\beta \mathrm{EGF}(10 \mathrm{ng} / \mathrm{ml})$. CD133-CD44-negative cells were cultured in DMEM/F12 medium with fetal calf serum. Cells were cultured at $37^{\circ} \mathrm{C}$, in a $5 \% \mathrm{CO}_{2}$ atmosphere with $95 \%$ humidity (Fig. 1).

Extraction of total cellular RNA. Total-RNA extraction was performed with TRIzol (Invitrogen, Carlsbad, CA, USA) according to the manufacturer's instructions. Extracted RNA samples were quantified by NanoDrop 1000 (Nanodrop, Wilmington, DE, USA). To remove any genomic DNA contamination, the samples were treated by DNase (DNA-free kit; Ambion, Austin, TX, USA).

miRNA chip analysis. The samples were analyzed with Human microRNA OneArray ${ }^{\circledR}$ v3 which is produced by Phalanx Biotech Group (Belmont, CA, USA). Human microRNA OneArray microarrays are made of polydeoxynucleotide probes spotted onto a proprietary chemical layer coated on top of a 1"x3" (25x75 mm) standard format microarray glass slide. Each probe is spotted onto the array in a highly consistent manner using proprietary, non-contact spotting technology. Each microarray contains 1711 unique human miRNA probes and 189 experimental control probes. Each unique probe has 3 features, and probes contain 100\% of Sanger miRBase v17 miRNA content. We used ULS miRNA labeling kit (Kreatech, Durham, NC, USA) to label target. We used miRNA OneArray
Hyb Buffer V3 and miRNA OneArray Hybridization Buffer II to complete the hybridization process. All these steps were conducted in accordance with the manufacturer's instructions. We used an Axon 4000B scanner (Molecular Devices, Sunnyvale, CA, USA) to scan miRNA chip, and GenePix 4.1 data analysis software.

$m R N A$ chip analysis. We analyzed samples with Illumina ${ }^{\circledR}$ Whole-Genome Gene Expression Direct Hybridization Assay system (direct hybridization assay) which integrates Illumina proprietary BeadArray technology, a precise microarray scanning system (the Illumina HiScan ${ }^{\mathrm{TM}}$ or iScan System or the Illumina BeadArray ${ }^{\mathrm{TM}}$ Reader), hybridization equipment and accessories, and standard, off-the-shelf sample labeling protocols. First we amplified RNA samples with Illumina ${ }^{\circledR}$ TotalPrep RNA Amplification kit. The procedure consists of reverse transcription to synthesize first strand cDNA, second strand cDNA synthesis, cDNA purification, in vitro transcription to synthesize cRNA and cRNA purification. All steps followed the instruction manual. The BeadArray chip analysis and data analysis were performed by YiKe Co., Shanghai, China. All analyses were conducted in accordance with the manual.

Real-time polymerase chain reaction assay for miRNA. Total-RNA was extracted from cells with TRIzol (Invitrogen). Total-RNA was assessed by measuring the absorbance at $260 \mathrm{~nm}$. cDNA was synthesized using ImProm-II reverse transcriptase. With strand cDNA $(0.5 \mu \mathrm{l})$, forward and reverse primers (both $0.5 \mu \mathrm{l}$ ) and SYBR green supermix (12.5 $\mu \mathrm{l}$ ), real-time qPCR was performed. Quantitative real-time PCR reaction was performed with the 7000 Sequence Detection System (ABI). Relative expressions were calculated using the formula $2^{-\Delta \Delta C T}$ values $\left(\Delta \mathrm{Ct}=\mathrm{Ct}^{\text {gene }}-\mathrm{Ct}^{\text {control }}\right)$. The primer sequences and PCR conditions are summarized in Table I.

miRNA target gene prediction. The target gene prediction software TargetScan was used to analyze differentially expressed miRNA (TargetScan human V6).

miRNA-mRNA correlation analysis. Negative regulation underlines the miRNA-mRNA relationship. We performed negative correlation analysis of significant expression patterns. We focused on the intersection of the 6444 miRNA target genes and the 2049 mRNA gene.

Gene ontology analysis of inversely related target genes. We first mapped target genes to each node of the gene ontology (GO) database. The analysis was carried out using the software DAVID (http://david.abcc.ncifcrf.gov/), according to the statistical test method (P-value) of significantly enriched categories ( $\mathrm{P} \leq 0.01$ as a result of the final output).

Pathway analysis of inversely related target genes. We first mapped target genes to the KEGG pathway database. We used the software DAVID for analysis (http://david.abcc.ncifcrf. gov/). $P \leq 0.05$ was regarded as statistically significant.

Network analysis of inversely related target genes

Functional regulatory network of miRNA target genes: the miRNA-GO-network. The target gene cluster was classified in 
A
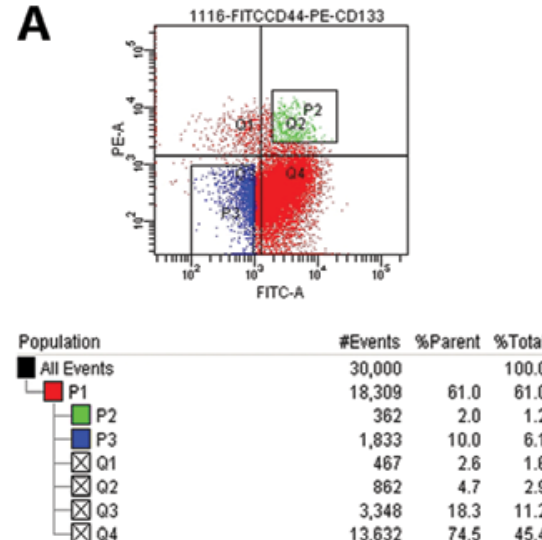

B

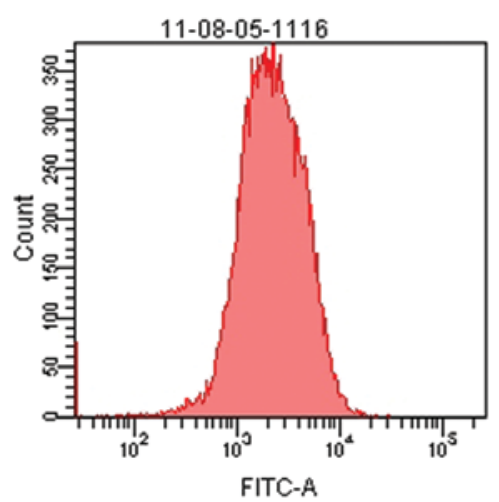

C

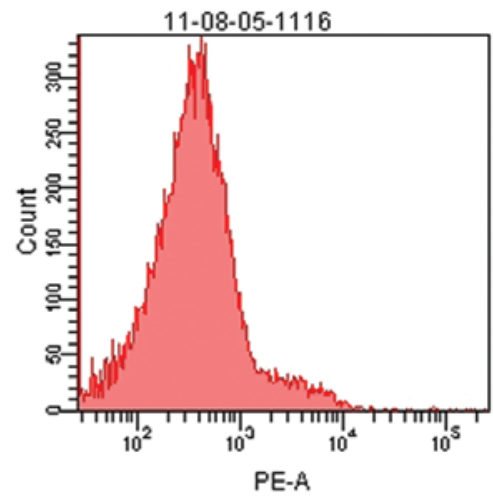

Figure 1. Isolation and characterization of colon stem cells in the SW1116 cell line. (A) CD44 was analyzed using FITC-labeled antibody. CD133 was analyzed using PE-labeled antibody. Top, methodology of flow cytometry cell sorting in the SW1116 cell line. P2, CD44 ${ }^{+}$CD133 ${ }^{+}$sorting area; P3, CD44-CD133- sorting area. P2 accounted for only $1.2 \%$ of the total number of cells; P3 accounted for only $6.1 \%$ of the total number of cells. (B and C) The expression of cell surface CD44 and CD133 antibody following amplification.

Table I. Sequences of primers and parameters for reverse-transcription and real-time PCR.

Forward primer (5'-3')

Reverse primer (5'-3')

\begin{tabular}{lll}
\hline MiR-29a & ACACTCCAGCTGGGTAGCACCATCTGAAAT & Kit provides \\
MiR-29b & ACACTCCAGCTGGGTAGCACCATTTGAAATC & Kit provides \\
MiR-449b & ACACTCCAGCTGGGAGGCAGTGTATTGTTA & Kit provides \\
MiR-4524a & ACACTCCAGCTGGGATAGCAGCATGAACCT & Kit provides \\
U6 & CTCGCTTCGGCAGCACA & AACGCTTCACGAATTTGCGT \\
NRAS & TGAAACCTCAGCCAAGACCAGACA & TGGCAATCCCATACAACCCTGAGT \\
FOS & TGTCTGTGGCTTCCCTTGATCTGA & TGGATGATGCTGGGAACAGGAAGT \\
WASF2 & AGATGCTGCAGGACACCAAGGATA & ACCAAAGTGGGTGGATACCCAGAA \\
COL5A1 & TGCTCCAGGGATTCCTTCAAGGTT & ATAGGAGAGCAGTTCCCACGCTT \\
CDK6 & TGCACAGTGTCACGAACAGACAGA & TTAGATCGCGATGCACTACTCGGT \\
CCND1 & AGAAGCTGTGCATCTACACCGACA & TGATCTGTTGTTCTCCTCCGCCT \\
E2F3 & AGTTCATTCAGCTCCTGAGCCAGT & CAGCCCATCCATTGGACGTTGTTT \\
GNG12 & AGCACCAACAATATAGCCCAGGCA & ACTCCTGGCATGTTCCTCACAGTA \\
GNA12 & TCAAGAAGCACTTCCCGGACTTCA & TTTCACAGCATGGAACACGAAGCG \\
$\beta$-actin & ACCAACTGGGACGACATGGAGAAA & TAGCACAGCCTGGATAGCAACGTA \\
\hline
\end{tabular}

accordance with the classification of the GO BP. miRNA-GO network was built. The network reflects the target miRNA target gene function. Network eigenvalue (degree) was calculated according to the location of each miRNA function. The miRNAs with the highest eigenvalues which regulate a number of gene functions and the sample status, are always located in pivotal positions in the network.

miRNA-gene network. Using the Sanger miRNA database, we screened target genes regulated by differentially expressed miRNAs (TargetScan). Then we took the differentially expressed genes and the intersection of target genes and differentially expressed genes, which is differential target genes regulated by differential miRNA. Classifying this part of differential target genes regulated by differential miRNA with GO BP, we found that significantly differential target genes belong to significant $\mathrm{GO}(\mathrm{P}<0.01)$. Using the relationship between miRNA and target gene, we built the miRNA-gene network.

Network of significant, differentially expressed target genes: miRNA-path network. With pathway analysis results, we built the pathway network. The network reflects the relationship between miRNAs and pathways. We calculated the specific degree according to the location of each miRNA and pathways in the network. miRNAs and pathways with the highest degree are always located in pivotal positions in the network. 


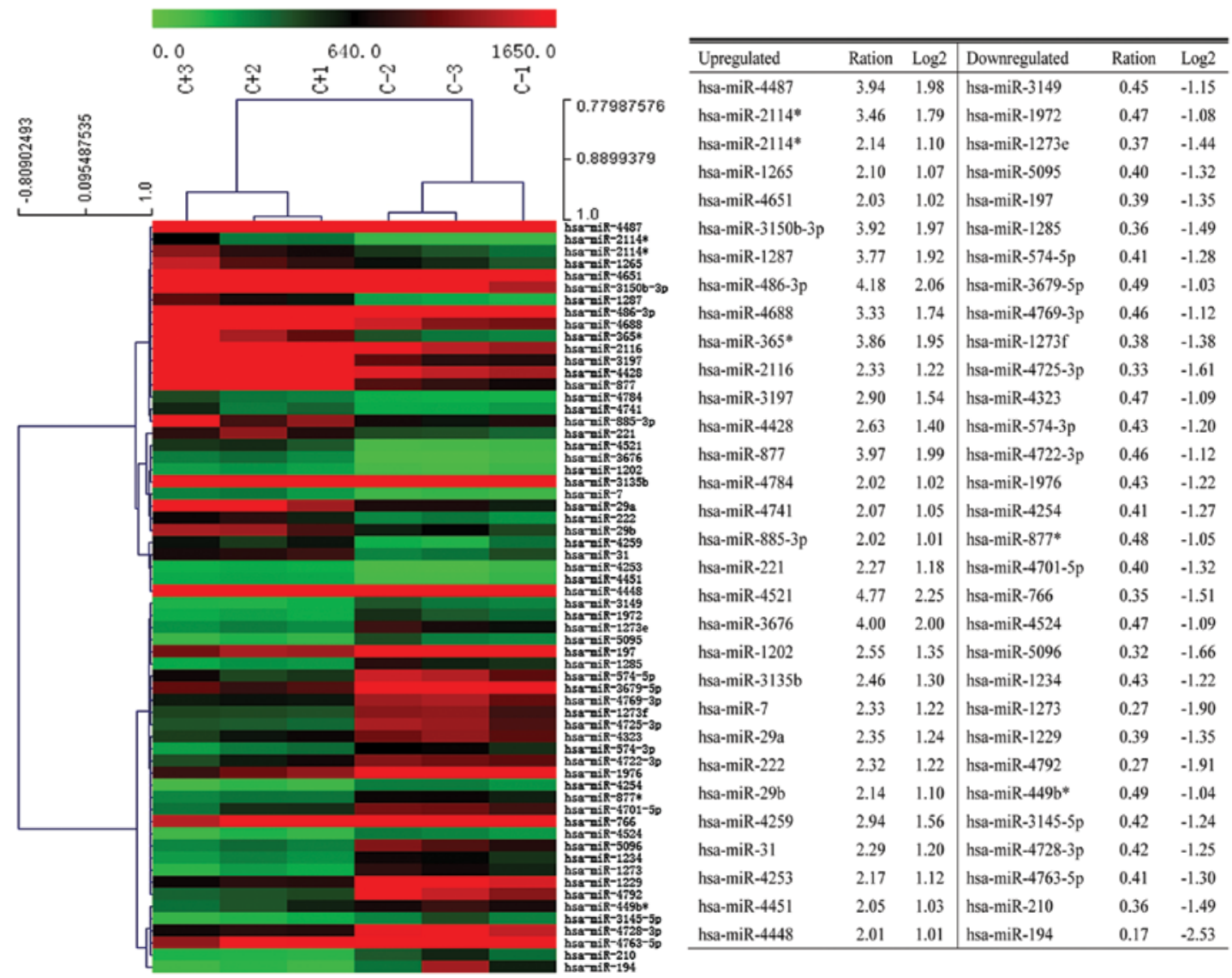

Figure 2. Differential miRNA profile expression in colon cancer stem and non-stem cells. All 31 upregulated and 31 downregulated miRNAs are listed. The degree of differentially expressed miRNAs compared to non-stem cells is listed in the table.

A

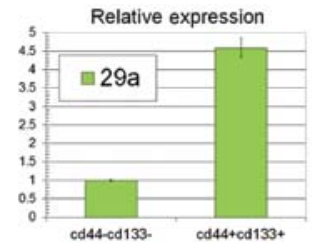

B
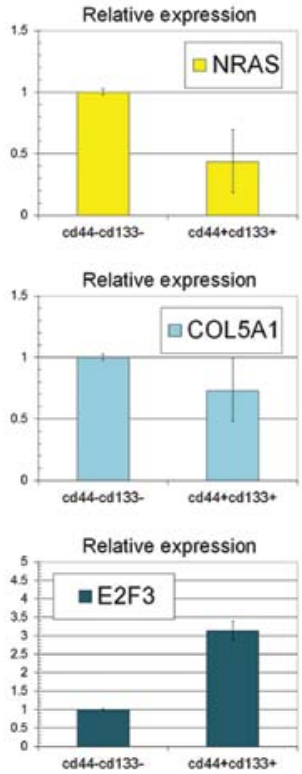

Relative Expression

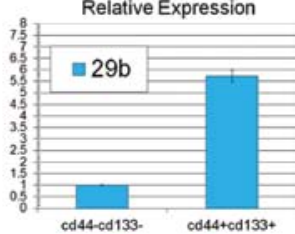

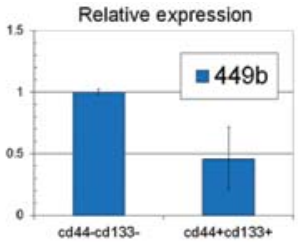
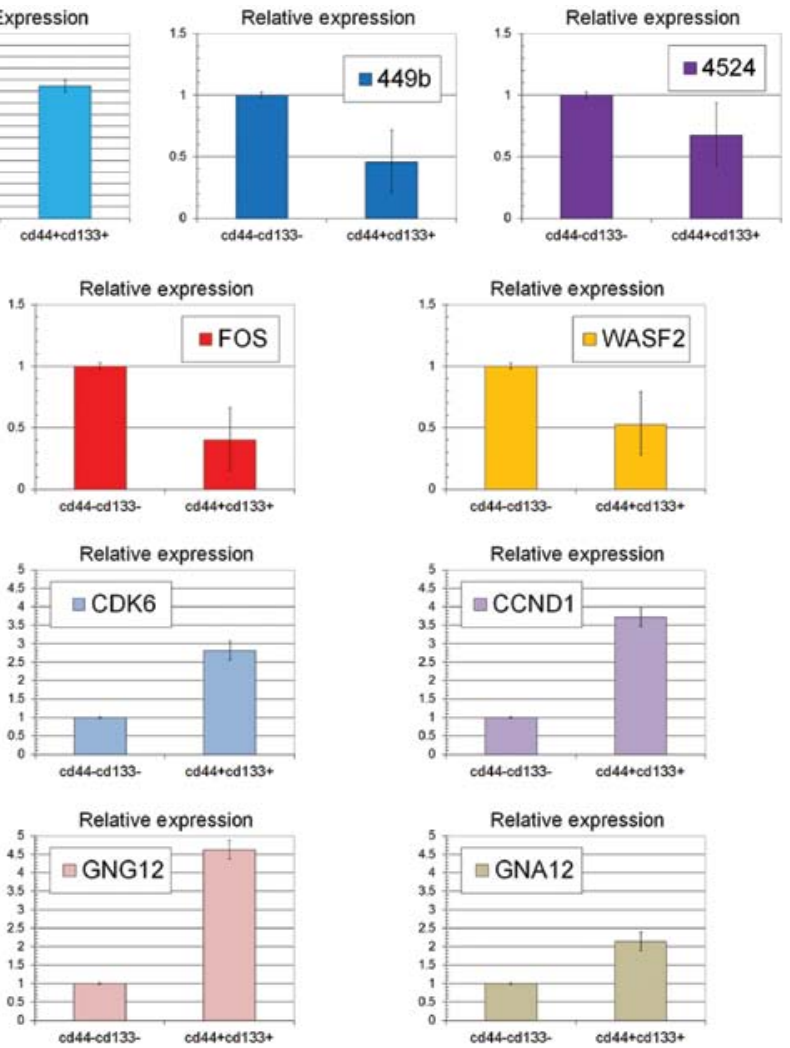

Figure 3. (A) The expression levels of miR29a, miR29b, miR449b and miR4524 were detected using RT-PCR. We found that miR29a and miR29b were upregulated while miR449b and miR4524 were downregulated in colon stem cells compared with non-stem cells $(\mathrm{P}<0.05)$. (B) The expression levels of NRAS, FOS, WASF2, COL5A1, CDK6, CCND1, E2F3, GNG12 and GNA12 were detected using RT-PCR. NRAS, FOS, WASF2 and COL5A1 were downregulated in colon stem cells $(\mathrm{P}<0.05)$. On the contrary, CDK6, CCND1, E2F3, GNG12 and GNA12 were upregulated $(\mathrm{P}<0.05)$. 


\section{Count of GOs in each node of the biological processes}

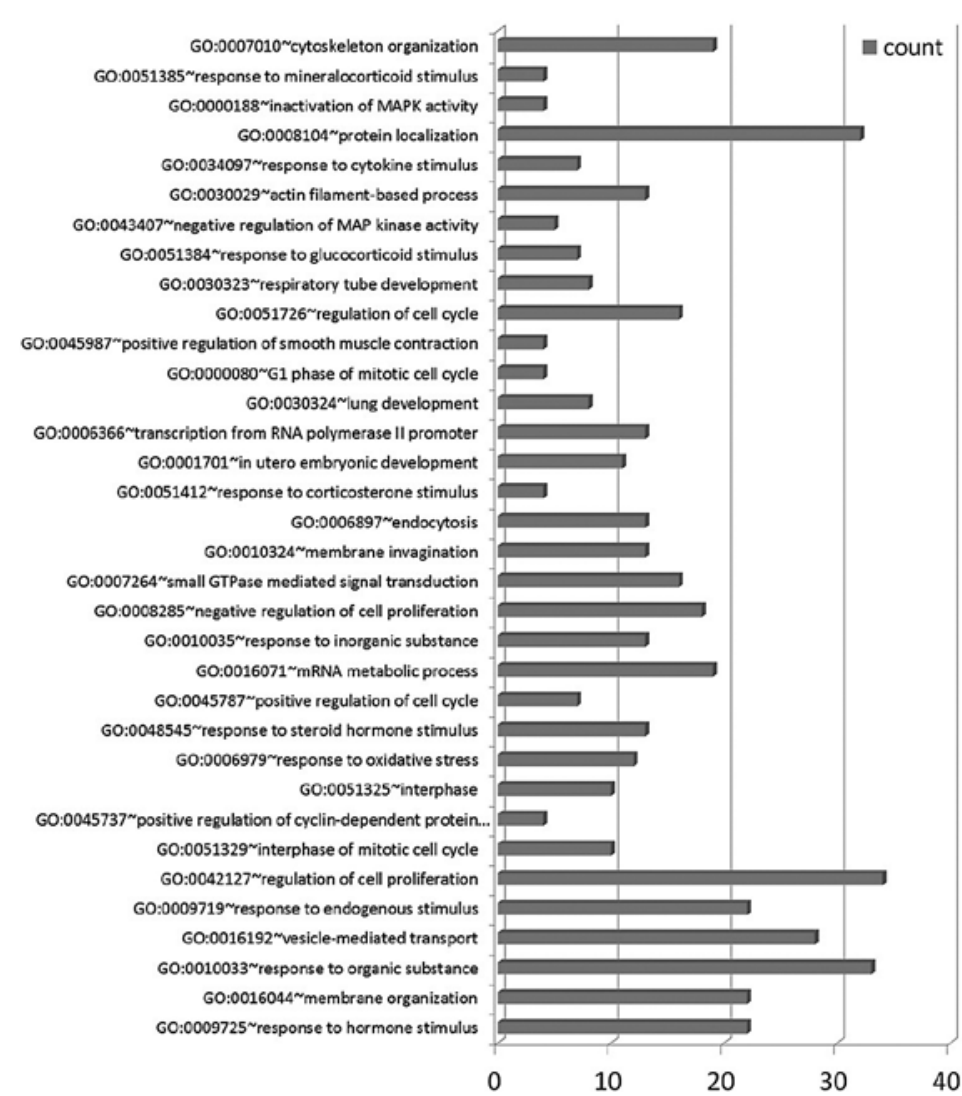

Count of GOs in each node of the molecular function

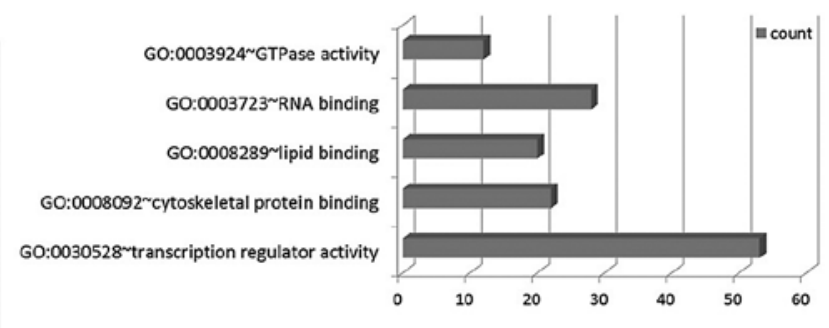

Count of GOs in each node of the cell components

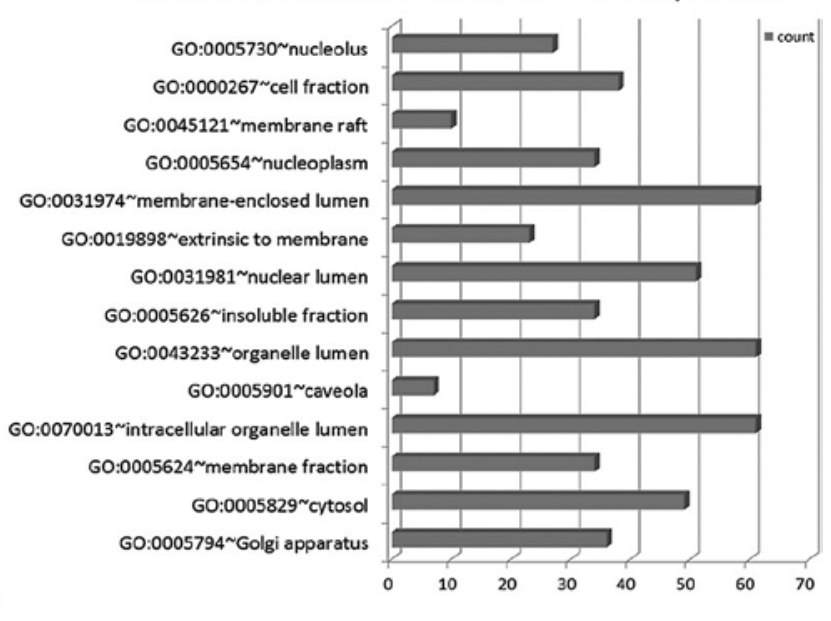

Figure 4. Differentially expressed mRNAs regulated by differentially expressed miRNAs involving a total of 34 significant GOs in the biological process, 5 GOs in molecular function and $14 \mathrm{GOs}$ in cellular component. Counts indicate enriched levels of genes acting on notes by the statistical test method (P $\leq 0.01)$.

\section{Results}

Differential miRNA expression profile in colon CSCs and nonstem cells. We used an array-based miRNA chip to investigate the differential miRNA expression profile in colon CSCs and non-stem cells. A total of 1711 human miRNAs were examined. There are 31 miRNAs significantly upregulated and 31 miRNAs significantly downregulated. Of these, miR-4521 was the most significantly upregulated miRNA. Similarly, miR-194 was the most significantly downregulated miRNA (Fig. 2).

Differential mRNA expression profile in colon CSCs and nonstem cells. We used mRNA chip to investigate the differential mRNA expression profile in colon CSCs and non-stem cells. We examined 34694 mRNAs. There are a total of 2049 differentially expressed mRNAs detected (Fig. 3).

GOs are regulated by miRNAs. We found that GOs were significantly regulated by differentially expressed genes in the biological process, molecular function and cellular component. Results show differentially expressed mRNAs regulated by differentially expressed miRNAs involving a total of 34 significant GOs in the biological process, 5 GOs in the molecular function and 14 GOs in the cellular component. The most differentially expressed GOs in the biological process are hormone stimulus, membrane organization, response to organic substance, vesicle-mediated transport, endogenous stimulus, regulation of cell proliferation, interphase of mitotic cell cycle, positive regulation of cyclin-dependent protein kinase activity and regulation of cell cycle. The most differentially expressed GOs in molecular function are transcription regulator activity, cytoskeletal protein binding, lipid binding, RNA binding and GTPase activity. The most differentially expressed GOs in cellular component are golgi apparatus, cytosol, membrane fraction and intracellular organelle lumen. Genes were classified in accordance with the GO biological process, and we constructed the miRNA-GO-network. miRNAs in different locations of the network have a different degree. The miRNA which has the highest degree is located in the central position of the network. From the network, we find that hsa-miR-29a, hsa-miR-29b, hsa-miR-449b, hsa-miR-4524 and hsa-miR-7 are involved in more GOs related to the characteristics of the sample. Regulation of cell proliferation, vesicle-mediated transport, response to organic substance and protein localization are important GOs represented by miRNA target genes (Figs. 4 and 5).

Signaling pathways are regulated by miRNAs. We used the KEGG database to analyze target genes. The target genes regulated by differentially expressed miRNAs involving a 


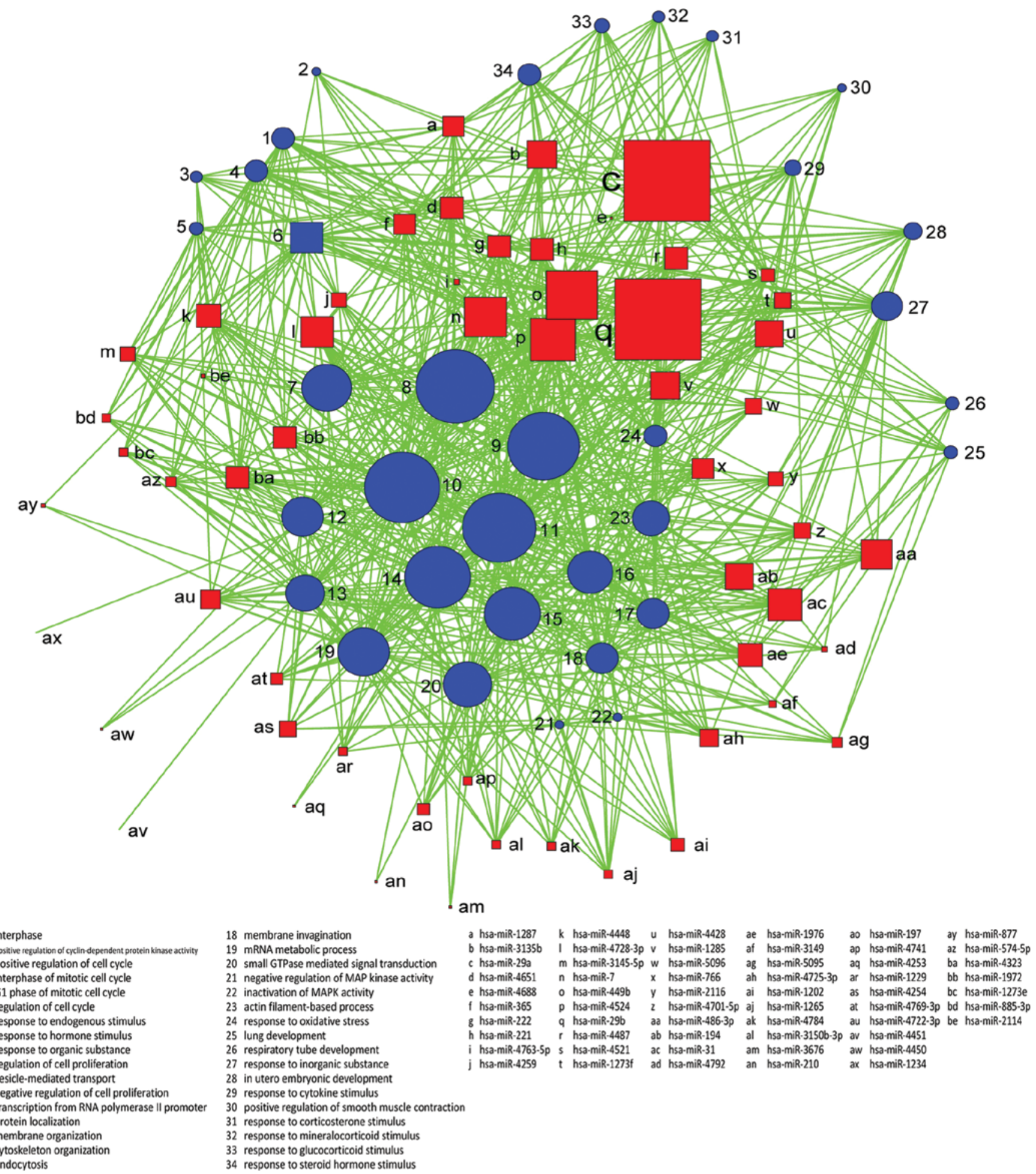

Figure 5. A network diagram showing the relationship between miRNAs and GOs. The rectangles represent miRNAs, the circles represent the GO functional annotation, a straight line indicates the regulation of relations between the miRNA and GO. The more GO miRNA was regulated, the greater its area; similarly, the more GO was regulated by miRNA, the greater its area.

total of 18 significant pathways, containing 47 genes. These pathways include pathways in cancer, endocytosis, regulation of actin cytoskeleton, the VEGF signaling pathway, the insulin signaling pathway, colorectal cancer and RNA degradation. Seen from the significant pathway relationship network, the main signal pathways are CRC, chronic myeloid leukemia, glioma, regulation of actin cytoskeleton, endocytosis and the VEGF signaling pathway, of which regulation of actin cytoskeleton and pathways in cancer have the largest degree. miRNA-29a, miRNA-449b, miRNA-29b, miRNA-4524, miRNA-194 are at the key position in the miRNA-pathwaynetwork. This is essentially the same as the result of the miRNA-GO-network (Figs. 6 and 7).

Regulation network of differentially expressed miRNAs. There are 62 miRNAs, 34 GOs, 18 pathways that have significant 


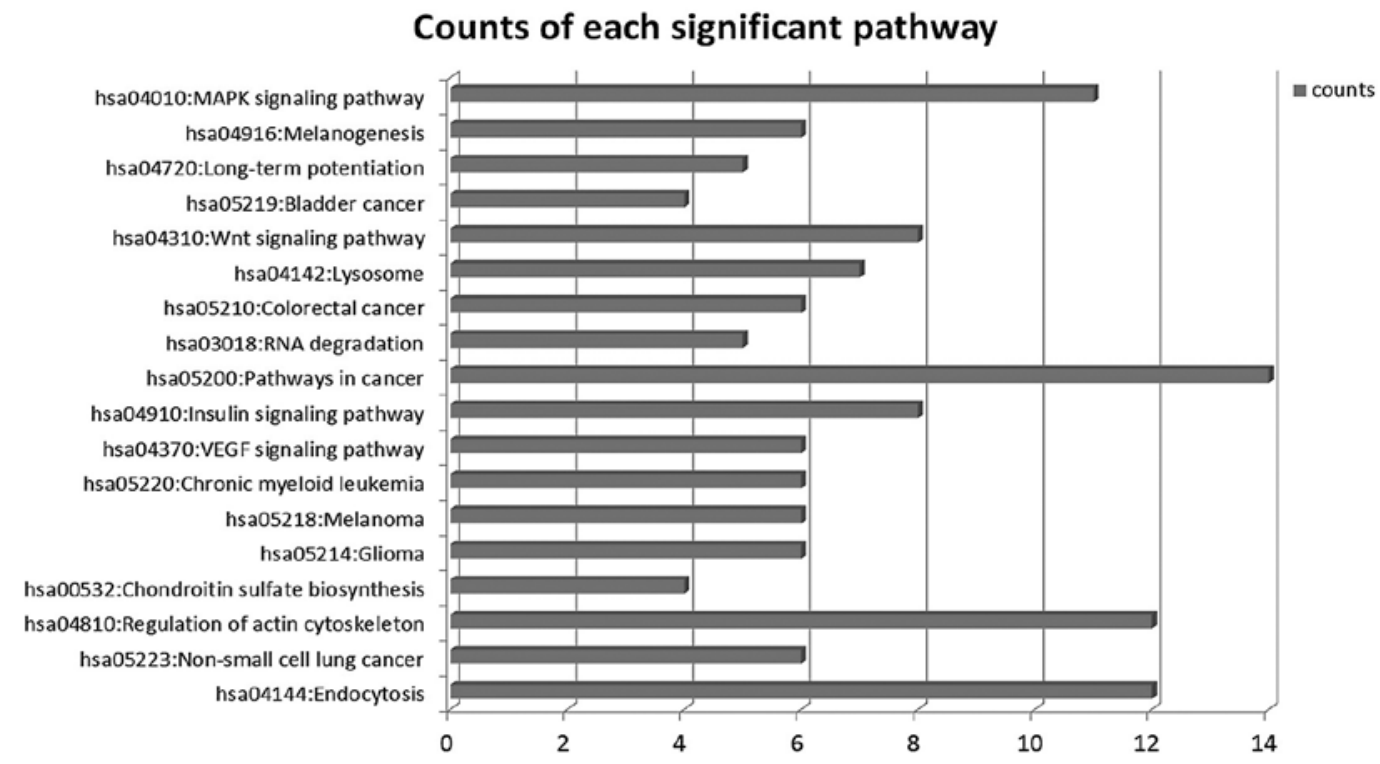

Figure 6. The target genes regulated by differentially expressed miRNAs involving a total of 18 significant pathways, containing 47 genes. Counts indicate enriched levels of pathways acting in keeping characteristics of colon stem cells by the statistical test method $(\mathrm{P} \leq 0.1)$.

1 Pathways in cancer

2 Regulation of actin cytoskeleton

3 Colorectal cancer

4 Chronic myeloid leukemia

5 Insulin signaling pathway

6 Endocytosis

7 Glioma

8 Melanoma

9 Non-small cell lung cancer

10 RNA degradation

11 VEGF signaling pathway

12 Chondroitin sulfate biosynthesis

\section{Counts of each significant pathway}




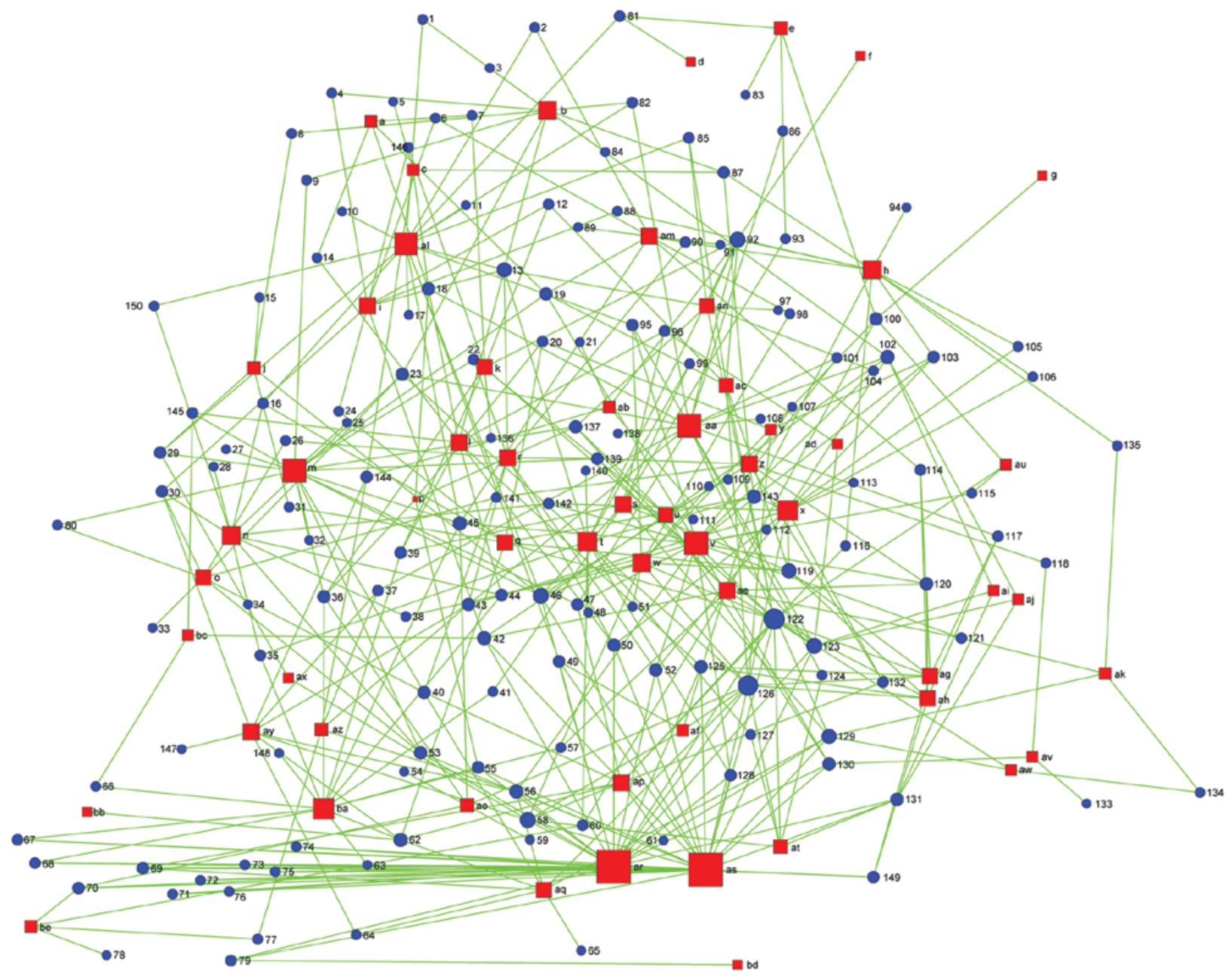

$\begin{array}{llllll}1 & \text { GMEB2 } & 16 & \text { CLASP1 } & 31 & \text { CTGF } \\ 2 & \text { SLC30A1 } & 17 & \text { RAB13 } & 32 & \text { CEBPA } \\ 3 & \text { PCGF2 } & 18 & \text { VPS37D } & 33 & \text { EZR } \\ 4 & \text { YBX2 } & 19 & \text { MYO5A } & 34 & \text { PPP3CB } \\ 5 & \text { CHMP4B } & 20 & \text { DUSP16 } & 35 & \text { SNCA } \\ 6 & \text { PALLD } & 21 & \text { XPO6 } & 36 & \text { EIF4EBP2 } \\ 7 & \text { FNBP1 } & 22 & \text { RBM12 } & 37 & \text { PACS1 } \\ 8 & \text { PTGS2 } & 23 & \text { VAMP2 } & 38 & \text { POLR2F } \\ 9 & \text { VAMP4 } & 24 & \text { DNAJC8 } & 39 & \text { IRS2 } \\ 10 & \text { CRK } & 25 & \text { LRP10 } & 40 & \text { ADCY6 } \\ 11 & \text { NUDC } & 26 & \text { OBFC2A } & 41 & \text { CNN3 } \\ 12 & \text { POM121C } & 27 & \text { TESK2 } & 42 & \text { FOXO4 } \\ 13 & \text { SSH2 } & 28 & \text { M6PR } & 43 & \text { CCND1 } \\ 14 & \text { PTGS1 } & 29 & \text { RBBP4 } & 44 & \text { BMPR2 } \\ 15 & \text { PRPF19 } & 30 & \text { SRF } & 45 & \text { TFRC }\end{array}$

$\begin{array}{lll}46 & \text { RAB6B } & 61 \\ 47 & \text { SPRED1 } & 62 \\ 48 & \text { TRPC4AP } & 63 \\ 49 & \text { SIX5 } & 64 \\ 50 & \text { DCP2 } & 65 \\ 51 & \text { TCF3 } & 66 \\ 52 & \text { XPO4 } & 67 \\ 53 & \text { COL5A1 } & 68 \\ 54 & \text { SPHK2 } & 69 \\ 55 & \text { KLF4 } & 70 \\ 56 & \text { GNG12 } & 71 \\ 57 & \text { PIM1 } & 72 \\ 58 & \text { NFIX } & 73 \\ 59 & \text { NSF } & 74 \\ 60 & \text { CYTH2 } & 75\end{array}$

NUP62
ATG7
OXR1
ENO2
VCP
SERP1
PAN2
CNOT8
LASP1
MCL1
TPM1
CPS1
CAV2
BTG2
LOX

ADA
PPP1CB
APLP1
NAPB
SHROOM3
NACC2
SWN1
PAPOLA
HMGB2
PAFAH1B1
NOTCH1
B4GALT7
HCFC1
NEK2
CHP

$\begin{array}{ll}91 & \text { FOXF1 } \\ 92 & \text { SAR1A } \\ 93 & \text { PPP2CA } \\ 94 & \text { HSPA4 } \\ 95 & \text { E2F3 } \\ 96 & \text { CALM1 } \\ 97 & \text { EIF2C2 } \\ 98 & \text { CXADR } \\ 99 & \text { SH3KBP1 } \\ 100 & \text { TCEB3 } \\ 101 & \text { FOSL1 } \\ 102 & \text { GRSF1 } \\ 103 & \text { HNRNPA1 } \\ 104 & \text { HNRNPD } \\ 105 & \text { AP1S1 }\end{array}$

$\begin{array}{llllll}106 & \text { RND3 } & 121 & \text { RAPGEF2 } & 136 & \text { MFGE8 } \\ 107 & \text { TUBGCP5 } & 122 & \text { SYNCRIP } & 137 & \text { RAB5B } \\ 108 & \text { PPP1R10 } & 123 & \text { AFF4 } & 138 & \text { LMAN2L } \\ 109 & \text { WIBG } & 124 & \text { SERPINE1 } & 139 & \text { SF3B3 } \\ 110 & \text { SLC25A36 } & 125 & \text { FOS } & 140 & \text { RAB31 } \\ 111 & \text { CAV1 } & 126 & \text { WASF2 } & 141 & \text { KHSRP } \\ 112 & \text { ARL2 } & 127 & \text { GDI1 } & 142 & \text { GNA12 } \\ 113 & \text { RAB10 } & 128 & \text { VPS37C } & 143 & \text { SLC9A1 } \\ 114 & \text { BCL2L11 } & 129 & \text { CDK6 } & 144 & \text { PXN } \\ 115 & \text { PRDM4 } & 130 & \text { AP4E1 } & 145 & \text { RABL3 } \\ 116 & \text { RBM3 } & 131 & \text { NRAS } & 146 & \text { NUPR1 } \\ 117 & \text { CDKN1C } & 132 & \text { SKAP2 } & 147 & \text { TPD52L1 } \\ 118 & \text { NQO1 } & 133 & \text { SIK1 } & 148 & \text { EGFR } \\ 119 & \text { HNRNPA3 } & 134 & \text { RAB35 } & 149 & \text { ROD1 } \\ 120 & \text { GNA13 } & 135 & \text { RNMT } & 150 & \text { NRP1 }\end{array}$

$\begin{array}{llll}\text { a } & \text { hsa-miR-4521 } & \text { p } & \text { hsa-miR-1229 } \\ \text { b } & \text { hsa-miR-4487 } & \text { q } & \text { hsa-miR-5095 } \\ \text { c } & \text { hsa-miR-4763-5p } & \text { r } & \text { hsa-miR-365 } \\ \text { d } & \text { hsa-miR-1234 } & \text { s } & \text { hsa-miR-4728-3p } \\ \text { e } & \text { hsa-miR-3149 } & \text { t } & \text { hsa-miR-486-3p } \\ \text { f } & \text { hsa-miR-3676 } & \text { u } & \text { hsa-miR-3145-5p } \\ \text { g } & \text { hsa-miR-4451 } & \text { v } & \text { hsa-miR-4524 } \\ \text { h } & \text { hsa-miR-4722-3p } & \text { w } & \text { hsa-miR-4448 } \\ \text { i } & \text { hsa-miR-4651 } & \text { X } & \text { hsa-miR-1976 } \\ \text { j } & \text { hsa-miR-1273f } & \text { y } & \text { hsa-miR-1265 } \\ \text { k } & \text { hsa-miR-766 } & \text { z } & \text { hsa-miR-4769-3p } \\ \text { I ha-miR-4428 } & \text { aa } & \text { hsa-miR-449b } \\ \text { m hsa-miR-31 } & \text { ab } & \text { hsa-miR-4792 } \\ \text { n hsa-miR-1285 } & \text { ac } & \text { hsa-miR-1202 } \\ \text { o } & \text { hsa-miR-5096 } & \text { ad } & \text { hsa-miR-4688 }\end{array}$

$\begin{array}{llll}\text { ae } & \text { hsa-miR-4323 } & \text { at } & \text { hsa-miR-4725-3p } \\ \text { af } & \text { hsa-miR-4741 } & \text { au } & \text { hsa-miR-877 } \\ \text { ag } & \text { hsa-miR-221 } & \text { av } & \text { hsa-miR-2114 } \\ \text { ah } & \text { hsa-miR-222 } & \text { aw } & \text { hsa-miR-1273e } \\ \text { ai } & \text { hsa-miR-4784 } & \text { ax } & \text { hsa-miR-4450 } \\ \text { aj } & \text { hsa-miR-3150b-3p } & \text { ay } & \text { hsa-miR-3135b } \\ \text { ak } & \text { hsa-miR-885-3p } & \text { az } & \text { hsa-miR-1287 } \\ \text { al hsa-miR-194 } & \text { ba } & \text { hsa-miR-7 } \\ \text { am hsa-miR-2116 } & \text { bb } & \text { hsa-miR-210 } \\ \text { an hsa-miR-197 } & \text { bc } & \text { hsa-miR-574-5p } \\ \text { ao hsa-miR-4701-5p } & \text { bd } & \text { hsa-miR-4253 } \\ \text { ap hsa-miR-4254 } & \text { be } & \text { hsa-miR-4259 } \\ \text { aq hsa-miR-1972 } & & \\ \text { ar hsa-miR-29a } & & \\ \text { as hsa-miR-29b } & & \end{array}$

Figure 8. A network diagram showing the relationship between miRNAs and genes. The rectangles represent miRNAs, the circles represent genes, the straight lines represent the regulation of relations between miRNAs and genes. The more the gene miRNA was regulated, the greater its area; the more the gene was regulated by miRNAs, the greater its area.

differences between colon CSCs and non-stem cells. Therefore, regulatory networks of differentially expressed miRNAs displaying the miRNAs with the highest degree affected the surrounding genes hsa-mi-29a, hsa-miR-29b, hsa-miR-4524, hsa-miR-449b, and hsa-miR-31 confirming previous results. Details are shown in Fig. 8. 


\section{Discussion}

Cancer microRNA (miRNA) expression profiling has been widely reported. Tumors of various organs in the body have corresponding miRNA expression profile changes. Therefore, miRNAs, as regulators of gene expression, are involved in the tumor development process, acting as oncogenes or tumor suppressor genes (17-20). Changes in miRNA expression profile are found at various stages of colon tumor development. Li et al reported that overexpression of miR-203 can significantly decrease cell proliferation and survival, and induce cell apoptosis in the p53-mutated colorectal cancer (CRC) cells (21). Strillacci et al demonstrated that downregulation of the miR-101 level could represent one of the leading causes of COX-2 overexpression in CRC cells (22). Li et al also reported that miR-181b can suppress proliferation of U87 glioma stem cells. Overexpression of miR-181b can reduce chemoresistance to temozolomide in U87 glioma stem cells (23). Therefore, these data show that miRNAs are related to the characteristics of tumor cells. Li et al recently found expression of breast cancer resistance protein BCRP/ABCG2 regulatory miRNAs (hsa-miR-328, -519c and -520h) in stem-like $\mathrm{ABCG}^{+}$cancer cells (24). Zhang et al identified a colon cancer stem cell (CSC) miRNA signature comprising a total of 19 differentially expressed miRNAs, such as miR-429, miR-155, and miR$320 \mathrm{~d}$, in the HT29 adenocarcinoma cell line (25). The above show that the special phenotype and biological characteristics of CSCs are results of miRNA regulation. However, differences in expression profiles of miRNA between colon CSCs and non-stem cells and the relationship between differential miRNA expression and the function of stem cells are rarely reported. Therefore, in our study, expression profiling of 1711 miRNAs based on OneArray microarray platform and 34694 mRNAs based on Illumina Whole-Genome system of samples of stem cells and non-stem cells of the SW1116 human CRC cell line was carried out to further explore the characteristics of CRC. The array data were confirmed using RT-PCR. We also classified colon stem cells with the profiling of miRNA. By defining miRNAs that are related to the characteristics of colon CSCs we will be able to further clarify the regulatory pathway and gain insight into the features of colon CSCs.

The results, by microarray analysis in the SW1116 CRC cell line, showed 62 miRNAs and 2049 mRNAs differentially expressed in colon stem cells compared to non-stem cells. Among these differentially expressed miRNAs, 31 miRNAs represented overexpression in colon stem cells, whereas the remaining 31 miRNAs demonstrated underexpression. Among these miRNAs, overexpression of mir-29a, mir-29b and underexpression of mir-449b, mir4524 were confirmed by quantitative RT-PCR assay, proving the chip results. This miRNA expression profiling indicates characteristics of colon CSCs. Thus, miRNA regulation is intricately related to distinctive features and the biological performance of colon CSCs.

Gene cluster controlling the cell cycle gene cluster is extremely important for the maintenance of stem cell growth and proliferation characteristics. GO analysis showed that there is a noticeable change (PPP1CB, CCND1, CDKN1C and CDK6) in cell cycle-related (GO: 0051329, interphase of mitotic cell cycle; GO: 0045787 , positive regulation of cell cycle; GO: 0000080, G1 phase of mitotic cell cycle; GO: 0051726, regulation of cell cycle) gene cluster. This shows that the change of cell cycle of stem cells is key to maintaining its important characteristics, and this distinguishes stem cells from non-stem cells. This feature gives stem cells their characteristics and their ability to proliferate and metastasize.

The change in the characterization of cell differentiation is an important feature of tumor stem cells that differentiates them from non-stem cells. Numerous studies have shown that the change of cell differentiation plays a crucial role in tumor occurrence, development and metastasis. We found that GO 0042127 (regulation of cell proliferation) related genes have significantly altered. Thirty-four genes correspond to this GO, including CAV1, KLF4, SERPINE1 and BMPR2. This reflects the need of the stem cells to maintain the level of the highdensity proliferation, in order to progress to distant metastases, cell proliferation-related gene expression was significantly changed.

Many signal transduction pathways in stem cells also changed. The most important change is the MAPK signaling pathway. MAPK, downstream signaling molecules with serine and threonine protein kinase activity in the Ras pathway, can format AP-1 acting on the nucleus to activate specific genes in order to pass the signal by activating the C-Fos, C-Jun transcription regulator. The MAPK signal transduction pathway plays an important role in stress responses such as inflammation and apoptosis. MAPK can promote endothelial cell proliferation and angiogenesis. Tumor angiogenesis can provide more nutrients to accelerate the growth of the tumor and to promote the proliferation of cancer cells. The MAPK pathway plays a unique role in the growth of the tumor stem cell and its transfer characteristics (26). The Wnt pathway is also involved. The majority of downstream target genes of the Wnt pathway is involved in cell proliferation and apoptosis genes. Playing a key role in the embryo during development, the Wnt pathway occurs with a variety of human tumors, especially in CRC. In colon CSCs, Wnt pathway-related genes (FOSL1, CCND1, CHP, FZD9 FZD4, PPP3CB PPP2CA, TBL1X) change (27). In addition, the Jak-STAT signaling pathway, ErbB signaling pathway, VEGF signaling pathway also showed varying degrees of change in colon CSCs.

Changes in cytoskeletal proteins may also be involved in stem cells. We found that the pathway of regulation of actin cytoskeleton is involved in a significant change. Kasper et al found that actin cytoskeleton is involved in mesenchymal stem cell aging (28). Cell-matrix adhesion is closely related to a variety of cellular processes such as cell migration, cell differentiation, and cell proliferation. Pathways such as focal adhesion, gap junction, adherens junction and tight junction are also involved.

In conclusion, by analyzing the difference of miRNA and mRNA expression in colon CSCs and non-stem cells, we found that miRNAs play an important role in the expression of stem cell characteristics. This study provides a new perspective on CRC metastasis and recurrence and the findings of this study may contribute to the treatment and diagnosis of CRC.

\section{Acknowledgements}

The authors are grateful for the support of the Central Laboratory and Department of Pathology of Huashan Hospital. 


\section{References}

1. Jemal A, Murray T, Ward E, et al: Cancer statistics. CA Cancer J Clin 59: 225-249, 2009.

2. Lengauer C, Kinzler K and Vogelstein B: Genetic instabilities in human cancers. Nature 396: 643-649, 1998.

3. Baylin S and Ohm J: Epigenetic gene silencing in cancer - a mechanism for early oncogenic pathway addiction? Nat Rev Cancer 6: 107-116, 2006.

4. Esteller M: Cancer epigenomics: DNA methylomes and histonemodification maps. Nat Rev Genet 8: 286-298, 2007.

5. Huang E and Wicha M: Colon cancer stem cells: implications for prevention and therapy. Trends Mol Med 14: 503-509, 2008

6. Pardal R, Clarke M and Morrison S: Applying the principles of stem-cell biology to cancer. Nat Rev Cancer 3: 895-902, 2003.

7. Tan B, Park C, Ailles L and Weissman I: The cancer stem cell hypothesis: a work in progress. Lab Invest 86: 1203-1207, 2006.

8. Chen Y, Hsu H, Chen Y, et al: Oct-4 expression maintained cancer stem-like properties in lung cancer-derived CD133-positive cells. PLoS One 3: e2637, 2008.

9. Gangemi R, Griffero F, Marubbi D, et al: SOX2 silencing in glioblastoma tumor-initiating cells causes stop of proliferation and loss of tumorigenicity. Stem Cells 27: 40-48, 2009.

10. Todaro M, Alea M, Stefano DA, et al: Colon cancer stem cells dictate tumor growth and resist cell death by production of interleukin-4. Cell Stem Cell 1: 389-402, 2007.

11. Bartel DP: MicroRNAs: genomics, biogenesis, mechanism and function. Cell 116: 281-297, 2004.

12. Gregory RI, Yan KP, Amuthan G, et al: The microprocessor complex mediates the genesis of microRNAs. Nature 432: 235-240, 2004

13. Bernstein E, Caudy A, Hammond SM and Hannon GJ: Role for a bidentate ribonuclease in the initiation step of RNA interference. Nature 409: 363-366, 2001.

14. Ambros V: The functions of animal microRNAs. Nature 431: 350-355, 2004

15. Meister G and Tuschl T: Mechanisms of gene silencing by double-stranded RNA. Nature 431: 343-349, 2004.
16. Peters L and Meister G: Argonaute proteins: mediators of RNA silencing. Mol Cell 26: 611-623, 2007.

17. Harfe BD, McManus M, Mansfield JH, Hornstein E and Tabin CJ: The RNaseIII enzyme Dicer is required for morphogenesis but not patterning of the vertebrate limb. Proc Natl Acad Sci USA 102: 10898-10903, 2005.

18. Murchison EP, Stein P, Xuan ZY, et al: Critical roles for Dicer in the female germline. Genes Dev 21: 682-693, 2007.

19. Wang Y, Medvid R, Melton C, Jaenisch R and Blelloch R: DGCR8 is essential for microRNA biogenesis and silencing of embryonic stem cell self-renewal. Nat Genet 39: 380-385, 2007.

20. Wienholds E, Koudijs MJ, Eeden F, Cuppen E and Plasterk R: The microRNA-producing enzyme Dicer1 is essential for zebrafish development. Nat Genet 35: 217-218, 2003.

21. Li J, Chen YX, Zhao JF, Kong FR and Zhang YD: miR-203 reverses chemoresistance in p53-mutated colon cancer cells through downregulation of Akt2 expression. Cancer Lett 304: 52-59, 2011.

22. Strillacci A, Griffonia C, Sansone P, et al: MiR-101 downregulation is involved in cyclooxygenase-2 overexpression in human colon cancer cells. Exp Cell Res 315: 1439-1447, 2009.

23. Li P, Lu XM, Wang YY, et al: MiR181-b suppresses proliferation of and reduces chemoresistance to temozolomide in U87 glioma stem cells. J Biomed Res 24: 436-443, 2010.

24. Li X, Pan YZ, Seigel GM, et al: Breast cancer resistance protein BCRP/ABCG2 regulatory microRNAs (hsa-miR-328, $-519 \mathrm{c}$ and $-520 \mathrm{~h})$ and their differential expression in stem-like ABCG2 ${ }^{+}$ cancer cells. Biochem Pharmacol 81: 783-792, 2011.

25. Zhang HL, Li WH, Nan FF, et al: MicroRNA expression profile of colon cancer stem-like cells in HT29 adenocarcinoma cell line. Biochem Biophys Res Commun 404: 273-278, 2011.

26. Seger R and Krebs EG: The MAPK signaling cascade. FASEB J: 726-735, 1995

27. Polakis P: Wnt signaling and cancer. Genes Dev 14: 1837-1851, 2000.

28. Kasper G, Mao L, Geissler S, Draycheva A, Trippens J, Kühnisch J, Tschirschmann M, Kaspar K, Perka C, Duda GN and Klose J: Insights into mesenchymal stem cell aging: involvement of antioxidant defense and actin cytoskeleton. Stem Cells 27: 1288-1297, 2009. 